\title{
Comparison of localized modifications of the contact potential difference induced by Focussed Ion Beam processing of $\mathbf{n}$ and $\mathbf{p}$ doped Silicon.
}

\author{
Marion A. Stevens-Kalceff ${ }^{* * *}$ and David P. Kruss ${ }^{*}$ \\ *School of Physics, University of New South Wales, Sydney, 2052 NSW Australia. \\ ** Electron Microscope Unit, University of New South Wales, Sydney, 2052 NSW Australia.
}

Scanning Probe Microscopy techniques provide three dimensional nanometer scale spatial resolution information about the structural, mechanical and chemical properties of surfaces and nanoscale materials. Kelvin Probe Microscopy (KPM) is a specialized Atomic Force Microscopy technique in which long-range Coulomb forces between a conductive atomic force probe and a specimen enable the electrical potential difference at the specimen surface to be characterized.[1] The measured surface potential profile may be associated the variation in contact potential difference at the surface and/ or with a charge distribution within the specimen. Information about the spatial extent and relative magnitude of this charge distribution can be deduced from the surface potential data.[2]

Focussed ion beam processing of materials used in semiconductor technology is of great importance in fundamental physics (scattering, defect microanalysis) and practical applications (nanomodification, depth resolved analysis, TEM sample preparation).[3] Focussed Ion Beam processed n (phosphorous) doped and $\mathrm{p}$ (boron) doped Silicon $<100>$ crystal wafer specimens with $5 \Omega \mathrm{cm}$ resistivity have been investigated. The Silicon specimens were each processed in a series of square 5 x $5 \mu \mathrm{m}^{2}$ patterns with a continuous normal incident $30 \mathrm{keV}, 1000 \mathrm{pA}$ focussed Gallium ion $\left(\mathrm{Ga}^{+}\right)$ beam in an FEI xP200 Focused Ion Beam system for increasing exposure times/ doses. The reproducible characteristic differences in the spatial distribution of the contact potential associated with the $\mathrm{Ga}^{+}$irradiated $\mathrm{n}$ and $\mathrm{p}$ doped Silicon have been measured using Kelvin Probe Microscopy using a Veeco/ Digital Instruments extended Dimension 3000 Scanning Probe Microscope. Irradiation/ implantation induces highly localized modification of the contact potential difference within the previously homogeneous silicon specimens.

In both $\mathrm{n}$ and $\mathrm{p}$ doped Silicon specimens the observations of implantation/ sputtering induced changes in the surface topography are consistent. During the initial stages of implantation, the irradiated Silicon is amorphized. This results in the highly localized expansion of the specimen surface. The amorphized region expands in height up to several nanometers at the surface for both $\mathrm{n}$ and $\mathrm{p}$ doped Silicon. In both cases further ion beam processing results in a raised rim of amorphized material which remains at the periphery of the milled/ sputtered volume and interfaces the implanted specimen with the surrounding undamaged $\mathrm{p}$ or $\mathrm{n}$ doped Silicon. This amorphized region has a high defect density. These defects may act as trapping centers and reduce the mobility of charge through the amorphized region. While the ion induced modification of the $n$ and $p$ doped specimens over the range of doses investigated is consistent, the induced surface contact potential difference and the evolution of the $\mathrm{Ga}+$ implantation induced modification is significantly different.

In $\mathrm{Si}, \mathrm{Ga}$ is a $\mathrm{p}$ type dopant. Implantation of $\mathrm{Ga}^{+}$into $\mathrm{n}$ doped $\mathrm{Si}$ introduces a localized (inactive amorphous) region in which the $\mathrm{n}$ type Silicon is essentially reversed doped as the concentration of $\mathrm{Ga}+\mathrm{p}$ type dopants exceeds $\mathrm{n}$ type dopants. Annealing processes are necessary to activate the dopants/ restore the crystalline structure. The $\mathrm{n}$ doped Silicon has excess mobile electrons which are 
attracted and electromigrate toward the highly localized $\mathrm{Ga}^{+}$implanted regions. A proportion of the mobile electrons from the $\mathrm{n}$ doped Silicon may be trapped by the amorphized defect layer which borders the milled regions. The boundary between this ion processed ( i.e Ga implanted, $p$ type) region and the $\mathrm{n}$ type surrounding material may form in effect a depletion region similar to the situation in an unbiased $p-n$ junction through which the electromigration of the electrons from the $n$ type material is minimal.

There is no depletion region in the case of the $\mathrm{Ga}^{+}$implanted $\mathrm{p}$ doped Silicon. As $\mathrm{Ga}+\mathrm{is}$ a $\mathrm{p}$ type dopant in Silicon, Ga+ ion beam processing adds to the dopant concentration. In the case of $\mathrm{p}$ type Silicon the ion beam induced modification of the contact potential is due to the different concentrations and type of dopant. The concentration of Gallium within the implanted region exceeds dopant level concentrations for higher dose rates and dynamic equilibrium between the implantation, sputtering and redeposition processes is ultimately achieved.

Surface contact potential differences of up to a few hundred $\mathrm{mV}$ are measured and are highly correlated with Electron Microprobe data of the Gallium implanted region. The physical processes associated with the observed induced contact potential variation are localized, dynamic, and are dependent on pre-existing and irradiation induced defect/ dopant concentrations. The observed potentials are associated with a number of complex competing physical processes including amorphization ion neutralisation, positive/secondary ion emission/ sputtering, ion implantation and charge trapping resulting in local modification of the dopant profiles. This investigation shows that ion beam processing of $\mathrm{n}$ and $\mathrm{p}$ doped Silicon can result in significantly different local modification of the chemical composition (dopant profile) and modification of the contact potential difference of the specimen via implantation and electromigration. This has implications for the processing of semiconductor materials in a Focussed Ion Beam system.

\section{References}

[1] Bonnell, D. A. Scanning Probe Microscopy and Spectroscopy: Theory, Techniques and Applications. Wiley-VCH. New York, 2001.

[2] Stevens-Kalceff, M. A. Microscopy and Microanalysis 10, (2004) 797-803.

[3] International Technology Roadmap for Semiconductors (2004) http://public.itrs.net

[4] Support from the Australian Research Council and the Electron Microscope Unit at University of New South Wales is gratefully acknowledged. Contact author: Marion.StevensKalceff@unsw.edu.au 